\title{
PHYTOPHTHORA PALMIVORA, AGENTE DA PODRIDÃO DE RAIZ E FRUTOS DE MAMOEIRO NO ESTADO DE ALAGOAS ${ }^{1}$
}

\author{
JULIANA PAIVA CARNAÚBA², MÁRCIO FÉLIX SOBRAL³, DANIELA CAVALCANTI DE MEDEIROS FURTADO4, \\ IZAEL OLIVEIRA SILVA ${ }^{5}$, KIRLEY MICHELLY MARQUES DA SILVA ${ }^{6}$, EDNA PEIXOTO DA ROCHA AMORIM ${ }^{7}$
}

\begin{abstract}
RESUMO - O mamoeiro (Carica papaya) é uma das mais importantes fruteiras tropicais, e seus frutos contribuem com uma produção de 1,65 milhão de toneladas por ano para o Brasil. Um isolado de Phytophthora sp. obtido de frutos de mamoeiro da cultivar Havaí, em Alagoas, foi caracterizado morfologicamente e sua patogenicidade foi confirmada em frutos e mudas deste hospedeiro. Em meio suco V-8, os esporangióforos apresentaramse delgados, simples ou pouco ramificados, enquanto os esporângios se apresentaram ovóides a elipsóides, proeminentemente papilados e um pedicelo curto na porção basal, medindo em média 37,68 x 27,52 $\mu \mathrm{m}$. Através das características apresentadas pelo isolado e os sintomas induzidos, o agente causal foi identificado como Phytophthora palmivora. Apesar de essa doença já ocorrer em outros estados, como Bahia, Espírito Santo, São Paulo, Pernambuco e Pará, este é o primeiro relato de Phytophthora palmivora em mamoeiro no Estado de Alagoas.
\end{abstract}

Termos para Indexação: Carica papaya, etiologia, ocorrência. ${ }^{1}$

\section{PHYTOPHTHORA PALMIVORA, CAUSAL AGENT OF ROOT AND FRUIT ROTS OF PAPAYA IN THE STATE OF ALAGOAS, BRAZIL}

\begin{abstract}
The papaya (Carica papaya) is one of the most important tropical fruit crops and its fruit contribute with a production of 1.65 millions tons/year in Brazil. An isolated of Phytophthora sp. gotten from papaya fruits cv. Hawaii, in the state of Alagoas, was characterized morphologically and its pathogenicity to this fruits and seedling was confirmed. In a V-8 medium, the sporangiophores presented to be slim, simple or little ramified, while the sporangia presented ovoid to ellipsoid, prominently papillae and one short pedicel in the basal portion, measuring in average $37,68 \mathrm{x} 27,52$ (m. Through the characteristics presented by the isolated and the induced symptoms, the causal agent was identified as Phytophthora palmivora. Despite of this disease already occurring in other states like in Bahia, Espirito Santo, São Paulo, Pernambuco and Pará, this is the first report of Phytophthora palmivora on papaya in the state of Alagoas, Brazil.
\end{abstract}

Index terms: Carica papaya, etiology, occurrence.

O mamoeiro (Carica papaya L.) é uma das mais importantes fruteiras tropicais, e seus frutos contribuem com uma produção de 1,65 milhão de toneladas por ano para o Brasil (FAO, 2004).

A podridão das raízes e dos frutos do mamoeiro é considerada uma das principais doenças da cultura. No Brasil, não há estatísticas a respeito, mas perdas de frutos da ordem de 7 - 10\% têm sido relatadas (Liberato et al., 1993; Silva et al., 2001).

A doença está disseminada por quase todas as regiões produtoras e com o agravante de que as cultivares comerciais não apresentam resistência a essa enfermidade. O problema é ainda maior pelo fato de o seu agente etiológico afetar cerca de 80 espécies de plantas, entre as quais citros, cacaueiro, coqueiro e abacaxizeiro (Embrapa, 2000).

Em junho de 2005, em uma propriedade produtora de mamão, situada no município de São Miguel dos Milagres, em Alagoas, constatou-se a ocorrência de podridão dos frutos de mamoeiro da cultivar Havaí, na fase terminal de maturação. Os frutos apresentavamse cobertos com uma massa esbranquiçada com aspecto cotonoso, constituída de micélio do fungo. O objetivo deste trabalho foi identificar o agente causal da podridão dos frutos em plantas de mamoeiro.

Para o isolamento de Phytophthora sp., empregou-se o meio de batata-dextrose-ágar (BDA) contendo inibidores seletivos (Masago et al., 1977). As culturas foram incubadas a $25^{\circ} \mathrm{C}$ no escuro, e posteriormente preservadas em água destilada estéril à temperatura ambiente.

A patogenicidade do isolado foi avaliada em frutos e em mudas de mamoeiro da cultivar Havaí, com 45 dias de idade. Em 10 frutos de mamão sadios, em fase de pré-maturação, inocularam-se discos de cultura pura, contendo as estruturas do fungo, sobre a superfície previamente desinfestada, enquanto nos frutos-testemunha utilizaramse discos contendo apenas meio de cultura (BDA). Os frutos foram submetidos a câmara úmida por 48 h.
Dez mudas cultivadas em solo autoclavado foram inoculadas conforme metodologia descrita por Sandler et al. (1986). Para manter a umidade, colocaram-se os vasos com nível de água de $+2 \mathrm{~cm}$ em recipiente plástico.

Os frutos apresentaram os sintomas iniciais de podridão no quarto dia após a inoculação (Figura 1A), enquanto nas mudas os sintomas de murcha surgiram no terceiro dia após a inoculação (Figura 1B). As culturas reisoladas em meio BDA contendo inibidores seletivos foram semelhantes às originais, confirmando a patogenicidade do isolado através do Postulado de Koch.

A identificação do agente causal foi realizada mediante estudos morfológicos e morfométricos dos esporangióforos e esporângios (Erwin \& Ribeiro, 1996). Os esporangióforos apresentavam-se delgados, simples ou pouco ramificados, enquanto os esporângios (Figura 1C) apresentavamse ovóides a elipsóides, proeminentemente papilados e um pedicelo curto na porção basal, medindo 43,16 - 26,56 x 31,54 - 19,92 $\mu$ m (37,68 x 27,52 $\mu \mathrm{m})$, estando de acordo com a literatura (Kimati et al., 1997).
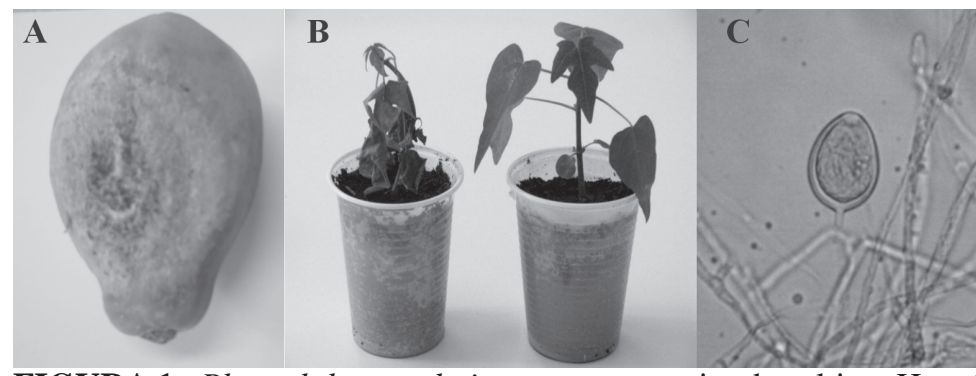

FIGURA 1 - Phytophthora palmivora em mamoeiro da cultivar Havaí. (A) Mamão inoculado com P. palmivora; (B) Sintomas de murcha três dias após a inoculação (esquerda) e testemunha (direita); (C) Esporângio de P. palmivora.

\footnotetext{
${ }^{1}$ (Trabalho 153/2005). Recebido: 29/09/2005. Aceito para publicação: 30/03/2006. Parte da dissertação de mestrado do $1^{\circ}$ autor;

2, 4 Estudante de Mestrado em Agronomia, CECA-UFAL/ Bolsista CAPES;

${ }^{3}$ Estudante de Agronomia, CECA-UFAL/ Bolsista PIBIC/CNPq;

${ }^{5,6}$ Estudante de Mestrado em Agronomia, CECA-UFAL/ Bolsista Fapeal;

${ }^{7}$ Prof ${ }^{a}$. Adjunta - Universidade Federal de Alagoas, Centro de Ciências Agrárias, Departamento de Fitotecnia e Fitossanidade, CEP 57100-000, Rio Largo, AL. Email: jcarnauba@hotmail.com.
} 
Através das características apresentadas pelo isolado, e os sintomas induzidos, o agente causal foi identificado como Phytophthora palmivora. Apesar de essa doença já ocorrer em outros estados, como Bahia e Espírito Santo (Liberato et al., 1993), São Paulo e Pernambuco (Reis, et al., 1997) e Pará (Trindade \& Poltronieri, 2002), este é o primeiro relato de $P$. palmivora em mamoeiro no Estado de Alagoas.

\section{REFERÊNCIAS}

EMBRAPA - Empresa Brasileira de Pesquisa Agropecuária. Mamão fitossanidade. Brasília; 2000. 91 p. (Embrapa Comunicação para Transferência de Tecnologia, Frutas do Brasil).

ERWIN, D.C.; RIBEIRO, O.K. Phytophthora diseases worldwide. St. Paul: APS Press, 1996. 562p.

FAO. FAOSTAT - Agricultural statistics database. Rome. World Agricultural Information Center. 2004. Disponível em: < http:// apps.fao.org.> Acesso em: 04 set. 2005.

KIMATI, H.; AMORIM, L.; BERGAMI, A. Filho.; CAMARGO, L.E.A.; REZENDE, J.A.M. Manual de fitopatologia: doenças das plantas cultivadas. 3.ed. São Paulo: Agronômica Ceres, 1997. v.2, 725p;

LIBERATO, J.R.; VANETTI, C.; RODRIGUES, C.H.; DIAS, V.P. Ocorrência de podridão de Phytophthora em mamoeiro (Carica papaya L.) no estado do Espírito Santo. Fitopatologia Brasileira,
Brasília, v.18, p. 324. 1993.

MASAGO, H.; YOSHIKAWA, M.; FUKADA, M.; NACANISHI, N. Selective inhibition of Pythium spp. On a medium for direct isolation of Phytophthora spp. From soils and plants. Phytopathology, St. Paul, v.67, n.3, p.425-428, 1977.

REIS, A.; MARIANO, R. L.R.; MICHEREFF, S.J.; MENEZES, M. Phytophthora palmivora, agente da podridão de raiz e frutos de mamão em Pernambuco. Fitopatologia Brasileira, Brasília, v. 22, n.4, p. 565-565, 1997.

SANDLER, H.A.; TIMMER, L.W.; GRAHAM, J.H. Timing of application of metalaxyl and fosetyl-aluminium for control of Phytophthora foot rot. Proceedings of the Florida State Horticultural Society, Winter Haven, v.99, p. 57-91, 1986.

SILVA, G.S. Podridão de raízes e dos frutos do mamoeiro. In: LUZ, E.D.M.N.; SANTOS, A.F.; MATSUOKA, K.; BECERRA, J.L. (Ed.). Doenças causadas por Phytophthora no Brasil. Campinas: Livraria e Editora Rural, 2001. p. 413-432.

TRINDADE, D.R.; POLTRONIERI, L.S. ; ALBURQUERQUE, F.C. Phytophthora palmivora causando podridão de frutos de mamoeiro no Estado do Pará. Fitopatologia Brasileira, Brasília, v. 27, n. 3, p. 388-388, 2002. 